\title{
Comment on the Paper: ‘Novel Approach to Estimate Kidney and Cyst Volumes Using Mid-Slice Magnetic Resonance Images in Polycystic Kidney Disease'
}

\author{
Cristiana Corsi $^{a}$ Renzo Mignani ${ }^{b}$ Dario Turco ${ }^{a}$ Riccardo Magistroni ${ }^{c}$ Stefano Severi ${ }^{a}$ \\ ${ }^{a}$ Department of Electrical, Electronic and Information Engineering 'Guglielmo Marconi', University of Bologna, \\ Cesena, ${ }^{b}$ Nephrology and Dialysis Unit, Infermi Hospital, Rimini, and 'Surgical, Medical and Dental Department of \\ Morphological Sciences Related to Transplant, Oncology and Regenerative Medicine, University of Modena and \\ Reggio Emilia, Modena, Italy
}

We recently read and appreciated the paper 'Novel approach to estimate kidney and cyst volumes using mid-slice magnetic resonance images in polycystic kidney disease' by Bae et al. [1]. The paper is very interesting and we recognize the intensive and very useful work the Consortium for Radiologic Imaging Studies of Polycystic Kidney Disease (CRISP) has conducted in the last years on this topic to develop methods to define a surrogate marker of the disease. Indeed, one of the most relevant results of CRISP was the evidence that magnetic resonance (MR) imaging is the best procedure for estimating changes in renal and cystic volume in autosomal dominant polycystic kidney disease over short followup periods.

As stated in the study [1], assessment of total renal and cystic volume is usually computed by manually tracing renal or cyst contours in a set of contiguous MR images and by summing the products of the area measurements and the slice thickness. We completely agree with the authors that measuring the area of every slice in such amounts of images is laborious, time-consuming and not applicable in routine clinical practice. For this reason we share the idea the authors had that a simplification of this computation to speed up the measuring process could have an impact on the clinical scenario. Their solution is based on the analysis of a limited number of MR images to approximate kidney and cyst volumes. To this regard, we strongly believe that using image processing techniques to drastically reduce processing time and fully exploit the information contained in the acquired dataset without applying any geometrical approximation and provide reliable volume estimates could be helpful for the computation of the parameters CRISP proved to be clinically important. The availability of good quality MR images, refined image processing algorithms and computational power makes this option completely feasible.

Indeed, some works proposing semiautomated or highly automated methods to quantify total kidney volumes in autosomal dominant polycystic kidney disease patients without using geometrical modeling and approximations have been published [2-4]. In particular, in a work that some of us coauthored [3], an extremely strong correlation $(r=0.99)$ was found between an automated measurement (requiring about
$1 \mathrm{~min}$ for each kidney) and the stereologybased observed kidney volume. We regret that it was not cited by Bae et al. [1]. As a preliminary proof of concept, we compared the performance of that method (TKV1) [3], adapted to work on coronal images, and the one proposed by Bae et al. [1] (TKV2) against the renal volumes derived by manually tracing renal contours in a small group of autosomal dominant polycystic kidney disease patients. Data were acquired following CRISP recommendations in 15 patients (volume range: $363-$ 1,794 and $425-2,316 \mathrm{ml}$ for the right and left kidneys, respectively). We found kidney volumes predicted by TKV1 corresponded even better than the measurements from TKV2 with the reference volumes (for the right and left kidneys, respectively: TKV1 $-\mathrm{r}^{2}=0.90$ and 0.97 , error $\mathrm{SD}=180$ and $158 \mathrm{ml}$; TKV $2-\mathrm{r}^{2}=0.86$ and 0.89 , error $\mathrm{SD}=218$ and $289 \mathrm{ml}$ ). These preliminary results support our hypothesis that the application of highly automated image processing techniques can provide a fast computation of reliable estimates. Regarding cyst volume quantification, the results showed by Bae et al. [1] are very promising since very few studies have been per-

\section{KARGER}

E-Mail karger@karger.com

www.karger.com/ajn (c) 2014 S. Karger AG, Base

0250-8095/14/0392-0163\$39.50/0
Cristiana Corsi, $\mathrm{PhD}$

Department of Electrical, Electronic and Information Engineering 'Guglielmo Marconi' University of Bologna, Via Venezia 52 IT-47023 Cesena (Italy)

E-Mail cristiana.corsi3@unibo.it 
formed for cyst volume quantification [5]. However, for the same aforementioned reasons, advanced image processing techniques should be taken into consideration to provide reliable estimates.

While not diminishing the contribution of this work, it seems important to emphasize the importance of other approaches based on image processing techniques which have already yielded good results and could be ready for clinical testing.

\section{References}

1 Bae KT, et al: Novel approach to estimate kidney and cyst volumes using mid-slice magnetic resonance images in polycystic kidney disease. Am J Nephrol 2013;38:333-341.

2 Grantham JJ, et al: Volume progression in polycystic kidney disease. N Engl J Med 2006; 354:2122-2130.

3 Mignani R, et al: Assessment of kidney volume in polycystic kidney disease using magnetic resonance imaging without contrast medium. Am J Nephrol 2011;33:176-184.
4 Cohen BA, et al: Intraobserver and interobserver variability of renal volume measurements in polycystic kidney disease using a semiautomated MR segmentation algorithm. AJR Am J Roentgenol 2012;199:387393

5 Bae K, et al: Segmentation of individual renal cysts from MR images in patients with autosomal dominant polycystic kidney disease. Clin J Am Soc Nephrol 2013;8:10891097. 